\title{
CARACTERIZAÇÃO DO ARCO LONGITUDINAL PLANTAR DE CRIANÇAS OBESAS POR MEIO DE ÍNDICES DA IMPRESSÃO PLANTAR*
}

\author{
CHARACTERIZATION OF THE LONGITUDINAL PLANTAR ARCH OF OBESE \\ CHILDREN USING PLANTAR PRINT INDEXES
}

Priscila S Souza*

Silvia $M$ A João**

Isabel de C N Sacco*****

\begin{abstract}
Souza PS, João SMA, Sacco ICN. Caracterização do arco plantar londtudinal plantar de crianças obesas por meio de índices da impressão plantar. Rev Bras Crescimento Desenvolv Hum 2007; 17(1):76-83.
\end{abstract}

Resumo: Objetivo: descrever o desenvolvimento do arco longitudinal medial (ALM) do pé de crianças obesas e verificar a concordância entre 5 índices da impressão plantar que caracterizam este arco. Métodos: 156 crianças obesas, ambos os sexos, idade entre 4 e 10 anos, pertencentes a escolas pública e privada da cidade de São Paulo, Brasil. As crianças classificadas como obesas foram selecionadas entre 1535 crianças estudantes de cinco escolas, com idade entre $4 \mathrm{e}$ 10 anos. Em seguida foram coletadas as impressões plantares de cada criança e calculados índices que caracterizaram o ALM: índice de Cavanagh e Rodgers - CR, índice de Staheli - IS1 e IS2, índice de Chipaux-Smirak ICS e ângulo alfa de Clarke - AA. Resultados: CR, IS e ICS mostraram que a grande alteração na forma do ALM ocorre na transição dos 8 para os 9 anos e o AA mostra dois momentos, passagem dos 6 para os 7 anos e dos 8 para os 9 anos. De uma maneira geral, a classificação IS1 foi a mais discrepante para classificar o ALM nessas crianças e o CR a mais constante. Conclusões: Observou-se que o ALM se forma mais tardiamente em crianças obesas ( 8 para 9 anos) em relação às crianças não obesas (5 para 6 anos). Quanto aos índices, houve semelhança na descrição do desenvolvimento do ALM entre CR, ICS e AA, sendo que o IS merece maior cuidado e restrição em seu uso.

Palavras-chave: Obesidade. Infância. Pé. Antropometria.

\section{INTRODUÇ̃̃O}

A obesidade infantil está crescendo no mundo todo e no Brasil há alguns poucos estudos sobre sua prevalência. Balaban e Silva ${ }^{1}$ relataram $35 \%$ de crianças com excesso de peso (sobrepeso e obesidade) em escolares de Recife. Abrantes et $\mathrm{al}^{2}$ afirmam que $11,9 \%$ e $8,2 \%$ das crianças entre 1 e 9 anos de idade no sudeste e no nordeste do Brasil, respectivamente, são obesas. Leão et $\mathrm{al}^{3}$ demonstraram prevalência de $15,8 \%$ de obesos em crianças entre 5 e 10 anos em Salvador e Anjos et al ${ }^{4}$, prevalência de $5 \%$ de obesos escolares no município do Rio de Janeiro. Esta condição leva

\footnotetext{
* Trabalho realizado no Laboratório de Biomecânica do Movimento e Postura, Depto. Fisioterapia, Fonoaudiologia e Terapia Ocupacional da Faculdade de Medicina da Universidade de São Paulo

** $\quad$ Fisioterapeuta formada pela Faculdade de Medicina da Universidade de São Paulo - Av. Dr. Arnaldo, 455.

*** Professora Doutora do Depto. Fisioterapia, Fonoaudiologia e Terapia Ocupacional da Faculdade de Medicina da Universidade de São Paulo.

**** Professora Doutora do Depto. Fisioterapia, Fonoaudiologia e Terapia Ocupacional da Faculdade de Medicina da Universidade de São Paulo. Lab. Biomecânica do Movimento e Postura Humana - Depto. Fisioterapia, Fonoaudiologia e Terapia Ocupacional - Faculdade de Medicina - USP. Rua Cipotânia, 51, Cidade Universitária - São Paulo - SP, Brasil, CEP: 05360-000. Tel: (11) 30917464; FAX: (11) 30917462. E-mail: icnsacco@usp.br
} 
a grandes alterações posturais, em coluna e membros inferiores principalmente, inclusive na estrutura dos pés infantis ${ }^{5,6}$. O desenvolvimento do pé envolve a formação de arcos plantares. Entre eles está o arco longitudinal medial (ALM) e alterações em sua forma alteram a funcionalidade do pé levando a complicações de toda a postura ${ }^{7}$. Crianças saudáveis apresentam um pé fisiologicamente plano, desenvolvendo seus arcos com o crescimento e desenvolvimento. Alguns estudos descrevem idades nas quais a maturação do ALM pode ocorrer. Magee ${ }^{8}$ considera que a partir dos 2 anos a forma do pé da criança é semelhante à do adulto. Volpon ${ }^{9}$ considera a fase de maior alteração do ALM entre 2 e 6 anos. Morioka et al ${ }^{10}$ e Hennig e Rosenbaum ${ }^{11}$ concluem que esta formação do ALM ocorre aos 6 anos. Donatelli ${ }^{12}$ afirma que apenas entre 6 e 8 anos o ALM poderá ser considerado maduro. Lin et al ${ }^{13}$ é mais generalista, afirmando que o ALM desenvolve-se na primeira década de vida. Crianças obesas, devido ao excesso de massa corpórea, podem apresentar mudanças na morfologia do pé, com rebaixamento ainda maior do ALM ${ }^{5,6}$.

Este estudo teve um delineamento transverso longitudinal e teve como objetivo descrever o desenvolvimento do ALM de crianças obesas de 4 a 10 anos de idade por meio de índices da impressão plantar: índice de Cavanagh e Rodgers CR ${ }^{14}$, índice de Staheli - IS1 ${ }^{15}$ e IS2 ${ }^{16}$, índice de Chipaux-Smirak - ICS ${ }^{17}$, o ângulo alfa de Clarke $-\mathrm{AA}^{18}$. Buscou-se comparar esses índices em cada grupo etário verificando se existe concordância entre eles na classificação deste arco.

\section{MÉTODOS}

Foram coletados os dados de massa e estatura de 1535 crianças com idade entre 4 e 10 anos em cada uma das 5 escolas amostradas por conglomerado. Então, foram calculados os valores de IMC e as crianças classificadas com IMC normal, com sobrepeso ou obesidade, segundo Cole et al ${ }^{19}$. Destas 1535 crianças cujo IMC foi calculado, $10,2 \%(n=156)$ foram consideradas obesas e selecionadas para análise no presente estudo. Os pais das crianças participantes assinaram termo de consentimento aprovado pela Comissão de Ética do Hospital Universitário da USP. A impressão plantar foi adquirida com a utilização de um pedígrafo, em descarga bilateral de peso e então os índices propostos neste estudo foram calculados.

O CR é obtido pela razão entre a área do médio pé e a área total do pé, excluindo-se os dedos, e classifica o ALM em baixo (CR $\geq 0,26)$, normal $(0,21<\mathrm{CR}<0,26)$ e alto $(\mathrm{CR} \leq 0,21)$. $\mathrm{O}$ IS é a razão entre a menor largura no mediopé e a maior largura no retropé e o IS 1 classifica o ALM em baixo (IS 20,88$)$, normal $(0,45<$ IS < $0,88)$ e elevado (IS $\leq 0,45)$ e o IS 2 classifica o ALM em plano (IS $\geq 0,9)$, rebaixado $(0,6 \leq$ IS $<$ $0,9)$, normal $(0,3 \leq$ IS $<0,6)$ e aumentado (IS < $0,3)$. O ICS é a razão entre a menor largura no mediopé e a maior largura no antepé e classifica o ALM em plano (ICS 20,45$)$, rebaixado $(0,4 \leq$ ICS $<0,45)$, intermediário $(0,3 £ \operatorname{ICS}<0,4)$, normal $(0,1 \leq \mathrm{ICS}<0,3)$ e elevado (ICS $<0,1)$. Por fim, o AA é o ângulo entre a tangente medial do pé e a reta que une o ponto mais medial do antepé e o ápice da curvatura do arco e classifica o ALM em baixo $\left(\right.$ AA $\left.<30^{\circ}\right)$, rebaixado $\left(30^{\circ} \leq\right.$ $\left.\mathrm{AA}<35^{\circ}\right)$, intermediário $\left(35^{\circ} \leq \mathrm{AA}<42^{\circ}\right)$, normal $\left(42^{\circ} \leq \mathrm{AA}<47\right)$ e elevado $\left(\mathrm{AA}>47^{\circ}\right)$.

Após verificação da normalidade dos dados pelo teste de Shapiro Wilks, foi utilizado o teste não paramétrico ANOVA Kruskal Wallis para comparação entre os valores absolutos de cada índice nos grupos etários e para a comparação entre os índices em cada grupo etário usou-se o teste ANOVA de Friedman, com escala ordinal (1-ALM diminuído, 2-ALM normal e 3-ALM elevado). Na comparação das proporções de ALM diminuído em cada índice, foi utilizado o Teste Qui-quadrado. Foi adotado nível de significância de $5 \%$.

\section{RESULTADOS}

A caracterização antropométrica da amostra está representada na tabela 1. Os valores absolutos médios dos índices por idade estão na tabela 2 com as respectivas comparações estatísticas e níveis descritivos (p). 
Tabela 1: Caracterização antropométrica da amostra de crianças obesas estudada

\begin{tabular}{|c|c|c|c|c|c|}
\hline Idade & $\mathbf{n}$ & Massa $(\mathbf{k g})$ & Estatura $(\mathbf{m})$ & IMC $\left(\mathbf{k g} / \mathbf{m}^{\mathbf{2}}\right)$ & Sexo Masculino $(\%)$ \\
\hline $\mathbf{4}$ & 13 & $24,7 \pm 2,3$ & $1,10 \pm 0,04$ & $20,9 \pm 1,6$ & 31 \\
\hline $\mathbf{5}$ & 13 & $28,7 \pm 3,0$ & $1,10 \pm 0,06$ & $21,8 \pm 1,6$ & 15 \\
\hline $\mathbf{6}$ & 18 & $31,6 \pm 4,5$ & $1,20 \pm 0,06$ & $21,6 \pm 1,0$ & 44 \\
\hline $\mathbf{7}$ & 24 & $38,5 \pm 5,9$ & $1,30 \pm 0,06$ & $23,5 \pm 2,0$ & 42 \\
\hline $\mathbf{8}$ & 23 & $43,4 \pm 6,7$ & $1,30 \pm 0,04$ & $23,5 \pm 1,8$ & 43 \\
\hline $\mathbf{9}$ & 45 & $44,3 \pm 11,4$ & $1,40 \pm 0,06$ & $22,4 \pm 3,3$ & 47 \\
\hline $\mathbf{1 0}$ & 20 & $51,3 \pm 13,0$ & $1,40 \pm 0,12$ & $25,2 \pm 3,3$ & 55 \\
\hline Total & 156 & $39,8 \pm 11,6$ & $1,30 \pm 0,12$ & $22,8 \pm 2,7$ & 42 \\
\hline
\end{tabular}

Tabela 2: Valores médios de cada índice do ALM por grupo de idade e os valores de p. (* representam diferenças estatisticamente significativas).

\begin{tabular}{|c|c|c|c|c|c|c|}
\hline Idade & CR & IS1 & IS2 & ICS & AA & p \\
\hline $\mathbf{4}$ & $0,31 \pm 0,10$ & $0,84 \pm 0,26$ & $0,84 \pm 0,26$ & $0,52 \pm 0,17$ & $28,2 \pm 12,5$ & $0,0001^{*}$ \\
\hline $\mathbf{5}$ & $0,28 \pm 0,06$ & $0,83 \pm 0,27$ & $0,83 \pm 0,27$ & $0,48 \pm 0,16$ & $27,2 \pm 11,4$ & $0,0007 *$ \\
\hline $\mathbf{6}$ & $0,29 \pm 0,05$ & $0,79 \pm 0,31$ & $0,79 \pm 0,31$ & $0,47 \pm 0,19$ & $29,4 \pm 13,9$ & $0,0032^{*}$ \\
\hline $\mathbf{7}$ & $0,29 \pm 0,05$ & $0,76 \pm 0,23$ & $0,76 \pm 0,23$ & $0,45 \pm 0,13$ & $34,9 \pm 12,2$ & $0,0000^{*}$ \\
\hline $\mathbf{8}$ & $0,29 \pm 0,05$ & $0,79 \pm 0,25$ & $0,79 \pm 0,25$ & $0,46 \pm 0,14$ & $34,1 \pm 12,0$ & $0,0000^{*}$ \\
\hline $\mathbf{9}$ & $0,25 \pm 0,07$ & $0,63 \pm 0,29$ & $0,63 \pm 0,29$ & $0,37 \pm 0,18$ & $40,2 \pm 13,3$ & $0,0000^{*}$ \\
\hline $\mathbf{1 0}$ & $0,25 \pm 0,04$ & $0,69 \pm 0,17$ & $0,69 \pm 0,17$ & $0,40 \pm 0,13$ & $40,0 \pm 10,5$ & $0,0000^{*}$ \\
\hline $\mathbf{p}$ & $0,0030 *$ & $0,0200 *$ & $0,0200^{*}$ & $0,0060 *$ & $0,0000^{*}$ & \\
\hline
\end{tabular}

Considerando a comparação entre os grupos de idade para cada índice, observou-se a existência de diferenças estatísticas significativas para todos os índices. Para o CR, os grupos 4, 5, 6, 7 e 8 anos foram estatisticamente iguais entre si, mas diferentes de 9 e 10, sendo estes também iguais entre si. Desta forma, entende-se como idade de "corte" para o desenvolvimento do arco para o índice de CR, 8 anos. No IS os grupos 4, 5, 6, 7 e 8 também foram iguais entre si e, com exceção do grupo 7 em relação ao 10, estes foram diferentes de 9 e de 10, sendo estes dois últimos iguais entre si. Da mesma forma, entende-se como idade de "corte" para o desenvolvimento do arco para o índice IS, 8 anos. Para o ICS, os grupos 4, 5, 6, 7 e 8 anos foram diferentes de 9 e de 10 anos, estes foram iguais entre si e houve uma diferença marginalmente significativa entre os grupos 4 e 7, os outros foram iguais entre si. Desta forma, entende-se como idade de "corte" para o desenvolvimento do arco para o índice ICS, 8 anos. Por fim, para o AA os grupos 4, 5 e 6 anos foram iguais entre si e estes foram diferentes de $7,8,9$ e 10 . O grupo de 7 anos foi igual ao de 8 e ambos diferentes de 9 e de 10 anos e, finalmente, estes iguais entre si. Desta forma, entende-se que há duas idades de "corte" para o desenvolvimento do arco para este índice, 6 e 8 anos, podendo-se considerar como média os 7 anos de idade.

Sendo assim, para todos os índices, os grupos de 4 a 6 anos foram sempre iguais. Para CR, IS e ICS a grande alteração na forma do ALM ocorre na transição dos 8 para os 9 anos e para o AA, há dois momentos, na passagem dos 6 para os 7 anos e dos 8 para os 9 anos. Em todos os índices observou-se que não há diferença entre os grupos 9 e 10, considerando não haver mudança no ALM nessa faixa etária.

Já na comparação entre as cinco classificações (CR, IS1, IS2, ICS e AA) por idade, observou-se diferença estatística em todos os grupos de idade. A classificação IS1 mostrou-se diferente de todas as outras em três dos sete grupos de idade (4, 7 e 9 anos). Nos outros quatro grupos de idade, o IS1 foi igual a apenas uma outra classificação. Aos 5 anos foi igual ao CR, aos 6,8 e 10 foi igual ao AA. O IS2 também se mostrou com diferença estatística em relação às outras muitas vezes e em todos os grupos de idade, sendo que aos 10 anos ela foi diferente das outras quatro classificações. Além disso, IS2 foi diferente de IS1 em todos os grupos de idade, mos- 
trando que realmente as duas classificações propostas para esse índice (IS) são bastante divergentes entre si. O AA destacou-se nos grupos 5, 8 e 9 , nos quais foi diferente de outras três classificações. Aos 5 anos o AA foi igual apenas ao IS2, aos 8 anos, igual ao IS1 e aos 9 anos, igual ao $\mathrm{CR}$, o que mostra uma diversidade importante e no grupo 6 o AA foi igual a todas as outras classificações. O CR foi sempre diferente do IS1, exceto aos 5 anos, foi sempre igual ao AA, exceto aos 8 anos e sempre igual ao ICS, exceto aos 9 anos. Além disso, foi diferente do IS2 apenas em três grupos (5,9 e 10 anos). Esse resultado mostra que o CR destacou-se entre os outros índices por ter apresentado muitas igualdades a outras classificações na maioria dos grupos de idade. Por fim, o ICS foi sempre igual ao CR, exceto aos 9 anos, como já citado, mas apresentou-se diferente em relação a outros índices em todos a os grupos, sendo sempre diferente de IS1. Portanto, de uma maneira geral, a classificação IS1 foi a mais discrepante para classificar o ALM nessas crianças em comparação às outras e o CR a mais constante. A tabela 3 descreve a classificação média do ALM por índice em cada grupo etário.

Quanto à proporção de ALM diminuído por índice nos grupos etários, ela foi maior nos grupos de 4 a 8 anos para todos os índices. $\mathrm{Na}$ comparação da incidência desse tipo de arco entre as idades por classificação, pôde-se observar que para todas as classificações houve diferença entre os grupos de 4 a 8 anos com os grupos de 9 e 10 anos. Para o CR, os grupos 4,6,7 e 8 foram diferentes dos grupos de 9 e 10 e estes foram diferentes entre si. Para o IS1, os grupos de 4 a 8 anos foram diferentes de 9 e de 10 , exceto o 7 , que se mostrou igual ao de 9 anos, não havendo diferença entre 9 e 10. Para o IS2 todos os grupos foram diferentes do 9, mas não do grupo 10. Para o ICS, os grupos de 4 a 8 anos foram diferentes de 9 e este foi igual ao de 10 anos. Por fim, para o AA, os grupos de 4 a 8 anos foram diferentes dos de 9 e 10 , exceto o grupo 8 , que se mostrou igual ao 10. Os valores das proporções estão descritos na tabela 4 .

Tabela 3: Classificação média do ALM por índice em cada grupo de idade.

\begin{tabular}{|c|c|c|c|c|c|}
\hline Idade & $\begin{array}{c}\text { Classificação CR } \\
\text { (Média) }\end{array}$ & $\begin{array}{c}\text { Classificação IS1 } \\
\text { (Média) }\end{array}$ & $\begin{array}{c}\text { Classificação IS2 } \\
\text { (Média) }\end{array}$ & $\begin{array}{c}\text { ClassificaçãoICS } \\
\text { (Média) }\end{array}$ & $\begin{array}{c}\text { ClassificaçãoAA } \\
\text { (Média) }\end{array}$ \\
\hline $\mathbf{4}$ & Baixo & Normal & Rebaixado & Plano & Baixo \\
\hline $\mathbf{5}$ & Baixo & Normal & Rebaixado & Plano & Baixo \\
\hline $\mathbf{6}$ & Baixo & Normal & Rebaixado & Plano & Baixo \\
\hline $\mathbf{7}$ & Baixo & Normal & Rebaixado & Plano & Rebaixado \\
\hline $\mathbf{8}$ & Baixo & Normal & Rebaixado & Plano & Rebaixado \\
\hline $\mathbf{9}$ & Normal & Normal & Rebaixado & Intermediário & Intermediário \\
\hline $\mathbf{1 0}$ & Normal & Normal & Rebaixado & Rebaixado & Intermediário \\
\hline
\end{tabular}

Tabela 4: Proporção de ALM (\%) diminuído por grupo de idade em cada índice.

\begin{tabular}{|c|c|c|c|c|c|}
\hline Idade & CR $(\%)$ & IS1 (\%) & IS2 (\%) & ICS (\%) & AA (\%) \\
\hline $\mathbf{4}$ & 77 & 42,3 & 88,5 & 96,2 & 88,5 \\
\hline $\mathbf{5}$ & 61,5 & 53,8 & 84,6 & 84,6 & 88,5 \\
\hline $\mathbf{6}$ & 69,5 & 44,5 & 77,8 & 86,1 & 72,2 \\
\hline $\mathbf{7}$ & 68,7 & 25 & 73 & 89,5 & 70,8 \\
\hline $\mathbf{8}$ & 74 & 34,7 & 78,2 & 89,1 & 67,3 \\
\hline $\mathbf{9}$ & 47,7 & 15,5 & 51,1 & 68,9 & 48,8 \\
\hline $\mathbf{1 0}$ & 42,5 & 7,5 & 72,5 & 85 & 50 \\
\hline
\end{tabular}




\section{DISCUSSÃO}

Foi possível obter o valor da incidência de crianças com excesso de peso nas escolas estudadas. Isso é de grande importância num momento em que se fala do rápido crescimento da obesidade no mundo todo, especialmente no Brasil. O número total de crianças de 4 a 10 anos pertencentes às escolas participantes foi de 1535. Dessas, 164 $(10,7 \%)$ apresentaram-se com sobrepeso para a idade, 156 (10,2\%) obesas. Assim, somam-se 320 (20,9\% do total) crianças com excesso de peso.

Todas as classificações (CR, IS1, IS2, ICS, AA) descreveram uma mudança na conformação do ALM durante o passar dos anos na infância de maneira semelhante. Existe uma formação gradual do ALM com o crescimento da criança, a qual pode ser resultado, segundo Staheli et al ${ }^{15}$ da diminuição do tecido gorduroso e da diminuição da frouxidão ligamentar, Sodré e Lourenço ${ }^{20}$ citam a melhora do equilíbrio pelo desenvolvimento músculo-esquelético e neural, que influencia a diminuição da área de apoio plantar, Viladot ${ }^{21}$ ainda acrescenta a maturação óssea na infância resultante dos esforços impostos aos pés. Entretanto, ao se tratar de crianças obesas, todo esse processo natural de formação do ALM apresenta-se atrasado em relação à população infantil em geral, já que se observou maior modificação no ALM dessas crianças na transição dos 8 para os 9 anos e, ainda, aos 10 anos, o arco não foi considerado maduro. A obesidade tem, então, grande influência na formação do ALM durante a infância, sendo essa apenas uma das inúmeras complicações no aparelho locomotor dessas crianças, como visto nos estudos de Hills et al ${ }^{22}$ e de Fisberg ${ }^{5}$. Com o rebaixamento ainda maior do ALM nessas crianças, pode-se esperar grandes alterações de alinhamento em membros inferiores e coluna por consequiência. De acordo com Sabiene e Minetti ${ }^{6}$, a biomecânica de todo o membro inferior apresenta-se alterada, interferindo na colocação dos pés em tarefas com descarga de peso e nas habilidades da locomoção. Hills e Parker ${ }^{23}$ afirmam que a marcha dessas crianças é mais assimétrica. Esse atraso pode ocorrer por inúmeros fatores relacionados aos processos responsáveis pela formação natural do ALM: a diminuição de tecido adiposo subcutâneo do pé que ocorre na infância ${ }^{15}$ pode estar ainda menor e mais atrasada no obeso, deixando o pé rebaixado por mais tempo. Outro fato relaciona-se com as maturações óssea e muscular, segundo Cailliet ${ }^{24}$ os ossos navicular e cuneiforme, que formam o ápice do arco, estão ainda moles e compressíveis e os músculos ainda não são capazes de sustentar carga na infância. Nos obesos, o excesso de peso pode interferir nessas maturações, já que provavelmente sobrecarrega ossos e músculos imaturos, prejudicando os processos naturais de desenvolvimento.

Estatisticamente, os índices classificaram o ALM de forma distinta em todos os grupos de idade, sendo o IS o índice que se mostrou diferente em relação aos outros quatro, tanto a classificação IS1, de Staheli et al ${ }^{15}$ quanto a IS2, de Echarri e Forriol ${ }^{16}$. Entretanto, ao considerarmos a classificação média do ALM para todos os índices (tabela 3), observou-se um padrão entre os índices $\mathrm{CR}$, ICS e AA. Este padrão foi a caracterização do arco de forma semelhante (plano) em todos os grupos de idade, exceto aos 9 e 10 anos, nos quais o CR já classifica o arco como normal, isto é, maduro. Isso pode ser justificado pelo fato do índice CR, entre os três aqui citados (CR, AA e ICS) apresentar apenas três classificações, baixo, normal e alto e os outros dois índices apresentarem cinco classificações, sendo três delas para arcos mais baixos, apresentando maior sensibilidade para detectar mudanças graduais no ALM, o que colabora para caracterização desse arco em crianças, tal como afirmam Morioka et al ${ }^{10}$, já que crianças apresentam um arco mais baixo que o fisiológico e mais importante ainda, em crianças obesas, nas quais o arco diminuído se perpetua por mais tempo, como visto no atual estudo. Em relação ao IS, Morioka et al ${ }^{10}$ propuseram a utilização de IS2 ao invés de IS1, já que esta apresenta valores relativamente altos para classificar o ALM em crianças e assim, muitas delas tiveram seu arco classificados como alto. No presente estudo, IS1 apresentou-se com a mesma limitação e classificou o ALM em todas as idades como normal, embora tenha detectado individualmente arcos planos e cavos nas idades, o que demonstra uma falta de sensibilidade para detectar o rebaixamento deste arco, esperado para 
essas crianças obesas. Entretanto, o IS2, mesmo sendo capaz de detectar o rebaixamento do ALM nas crianças obesas, não foi capaz de descrever as mudanças graduais de formação do mesmo, classificando-o da mesma forma em todas as idades.

Cabe aqui ressaltar que como cada índice utilizado é adquirido de maneira distinta, podese afirmar que se ocorrem alterações acentuadas em regiões específicas dos pés das crianças obesas, aquele índice que utiliza essa região em seu cálculo pode ser mais capaz de detectar tal alteração, como o caso do ICS. Este índice utiliza a maior largura do antepé em seu cálculo e nas impressões plantares deste estudo observou-se que os pés de crianças obesas apresentaram grande aumento na largura do antepé, o que fez com que o ICS apresentasse a maior incidência de pé plano quando comparado aos outros índices para todas as idades, exceto aos 5 anos.

Em relação à incidência de ALM baixo, foi observado que ela é maior nos grupos de idade mais baixa, estando em concordância com os estudos de Lin et al ${ }^{13}$ e de Echarri e Forriol ${ }^{16}$. Entretanto, se compararmos os valores do atual estudo com os descritos por Lin et al ${ }^{13}$, as incidências são maiores em todas as faixas etárias para todos os índices, o que é esperado por se tratar de uma pesquisa com crianças obesas, demonstrando mais uma vez a interferência do excesso de peso na formação do arco, atrasando esse processo e aumentando, então, sua ocorrência em crianças de idades nas quais não se espera arco baixo.

Conclui-se, então, que o ALM se forma mais tardiamente em crianças obesas, sofrendo as maiores alterações na passagem dos 8 para os 9 anos de idade, segundo os quatro índices estudados, o que demonstra atraso nesse desen- volvimento quando comparado a crianças não obesas, as quais apresentam maior alteração dos 5 para os 6 anos de idade. Entretanto, conservase a tendência de maturação desse arco. $\mathrm{O}$ atraso pode acontecer em função da sobrecarga exercida pelo excesso de peso sobre as estruturas formadoras do arco, como ossos e músculos imaturos ainda nas crianças ou mesmo por uma insensibilidade dos índices aqui estudados para detectar o desenvolvimento do ALM em um pé que apresenta um coxim gorduroso maior e mais persistente. Quanto às classificações, o CR é o mais citado e utilizado, reafirmando sua confiabilidade, entretanto exige uso de equipamento específico (planímetro) para seu cálculo, o que inviabilizaria em alguns centros de saúde. O ICS e o AA têm a vantagem de apresentarem dentro das suas cinco classificações do ALM, três para rebaixamento de arcos, fato importante para crianças, principalmente obesas, e desta forma permite maior precisão em sua aferição. O IS é o que merece maior cuidado e restrição em seu uso, pois qualquer uma das duas classificações propostas na literatura (IS1 e IS2) não foi sensível para detectar o desenvolvimento do arco nessas crianças e o IS1 nem mesmo detectou o rebaixamento deste arco.

\section{AGRADECIMENTOS:}

À Instituição Fapesp. Aos alunos, aos professores e aos coordenadores das escolas participantes: EMEI Maria José Galvão de França Pinto; EMEI Min. Synésio Rocha; Colégio Guilherme Dumont Villares; Colégio Pentágono Alphaville; MOVERE - Núcleo de Atividades Esportivas. À aluna Luciana Olcerenko Cicca, pelo auxílio em parte da coleta de dados. 


\begin{abstract}
Objectives - To describe the development of the medial longitudinal arch (MLA) of the foot of obese children and verify the concordance between 5 footprint indexes which characterize such arch. Methods: 156 obese children, both genders, between 4 and 10 years old, belonging to both public and private schools of the city of São Paulo, Brazil. The children were classified as obese according to Cole et al (2000) and selected among 1535 students between 4 and 10 years old from 5 different schools. Afterwards, the footprints of each obese child were collected and then the indexes that characterize the MLA were calculated: Cavanagh and Rodgers index - CR, Staheli index - IS1 and IS2, Chipaux-Smirak index - ICS, and footprint angle - AA. Results: CR, IS and ICS showed that a remarkable change in the MLA shape takes place in the transition from 8 to 9 years of age and the AA showed two transition ranges, from 6 to 7 and from 8 to 9 years of age. Generally speaking, the largest discrepancy was noticed in the IS1 classification when evaluating the MLA for those children and the CR one proved to be the most stable. Conclusion: It was observed that the MLA is formed later in obese children ( 8 to 9 years of age) comparing to non-obese children (5 to 6 years of age). Regarding the indexes, there was a similarity in the description of the MLA development between CR, ICS and AA. The IS needs more caution and its use should be restricted.
\end{abstract}

Key words: Obesity. Childhood. Foot. Anthropometry.

\section{REFERENCIAS}

1. Balaban G, Silva GAP. Prevalência de sobrepeso em crianças de uma escola da rede privada de Recife. Jornal de Pediatria. 2001; 77: 96-100.

2. Abrantes MM, Lamounier JA, Colosimo EA. Prevalência de sobrepeso e obesidade em crianças e adolescentes das regiões sudeste e nordeste. Jornal de Pediatria. 2002; 78(4): $335-40$.

3. Leão lscs, Araújo LMB, Moraes LTLP. Prevalência de obesidade em escolares de Salvador, Bahia. Arq Brás Endocrinol Metab. 2003; 47 (2): 151-7.

4. Anjos LA, Castro IRR, Engstrom EM, Azevedo AMF. Crescimento e estado nutricional em amostra probabilística de escolares no município do Rio de Janeiro, 1999. Cad Saúde Pública. 2003; 19 (supl 1): S171-9.

5. Fisberg M. Obesidade na Infância e na Adolescêcia. BYK, São Paulo, 1995.

6. Saibene F, Minetti AE. Biomechanical and physiological aspects of legged locomotion in humans. Eur J Appl Physiol. 2003; 88 (45):297-316.

7. Saltzman CL, Nawoczski DA, Talbot KDM. Measuremente of the Medial Longitudial Arch. Arch Phys Med Rehabil. 1995; 76 : 45-9.

8. Magee D J. Avaliação Musculoesuelética. Ed. Manole, São Paulo, 2002.
9. Volpon JB. Footprint analysis during the growth period. Journal of Pediatric Orthopaedics. 1994; vol.14, n.1, p.83-85.

10. Morioka EH, Onodera AN, Sacco ICN, Sá MR, Amadio AC. Avaliação do arco longitudinal medial através da impressão plantar em crianças de 3 a 10 anos [CDROM]. In: Anais do XI Congresso Brasileiro de Biomecânica, João Pessoa 2005.

11. Hennig EM, Rosenbaum D. Pressure distribution pattern under the feet of children in comparison with adults. Foot \& Ankle. 1995; 11(5), p 306-311.

12. Donatelli R, Wolf S L. The Biomechanics of the Foot and Ankle. Philadélphia, F A Davis Company, 1990 p.7-8.

13. Lin CJ, Lai KA, Kuan TS, Chou YL. Correlating factors and clinical significance of flexible flatfoot in preschool children. Journal of Pediatric Orthopaedics. 2001; 21: 378-382.

14. Cavanagh PR, Rodgers MM. The arch index: a useful measure from footprints. Journal of Biomechanics. 1987; 20(3), p.547-51.

15. Staheli LT, Chew DE, Corbett M. The longitudinal arch. The Journal of Bone and Joint Surgery. 1987 p. 426-428.

16. Echarri JJ, Forriol F. The development in footprint morphology in 1851 Congolese children from urban and rural areas and the relationship between this and wearing shoes. Journal of Pediatric Orthopaedics. 2003; 12-B (2), 141-146. 
17. Forriol F, Pascual J. Footprint analysis between three and seventeen years of age. Foot and Ankle. 1990; 11(2), p.101-104.

18. Clarke HH. An objective method of measuring the height of the longitudinal arch in foot examinations. Research Quartely. 1933; 4: 99-107.

19. Cole TJ, Bellizzi MC, Flegal KM, Dietz WH. Establishing a standart definition for child overweight and obesity worldwide: international survey. British Medical Journal. 2000; 320: 1240-3.

20. Sodré H, Lourenço AF. Propedêutica do
Tornozelo e Pé Infantil. In: Manual de Propedêutica Ortopédica, 8, UNIFESP, 1993.

21. Viladot A. Patologie del antepie. Toray, Barcelona. 1974.

22. Hills AP, Hennig EM, Byrne NM, Steele JR..The biomechanics of adiposity-structural and functional limitations of odesity and implications for movement. Obes Rev. 2002; 3(1):35-43.

23. Hills AP, Parker AW. Gait asymmetry in obese children. Neuro-Orthoped. 1991; 12: 29-33.

24. Cailliet R. Pé e Tornozelo. Síndromes Dolorosas. Editora Manole, São Paulo, 1977.

Recebido em 22/09/2006

Aprovado em 18/12/2006 